\title{
A Saline Tank Study of a Catheter Guiding Method for Ablative Therapy of Cardiac Arrhythmias by Application of an Inverse Solution to Body Surface Electrocardiographic Signals
}

\author{
D Kanai $^{1,2}$, Y Fukuoka $^{1}$, O Oyama ${ }^{2}$, AA Armoundas ${ }^{3}$ \\ ${ }^{1}$ Tokyo Medical and Dental University, Chiyoda, Tokyo, Japan \\ ${ }^{2}$ Meiji University, Kawasaki, Kanagawa, Japan \\ ${ }^{3}$ Massachusetts General Hospital, Boston, MA, USA
}

\begin{abstract}
This study investigates in a saline tank study the effectiveness of an inverse algorithm designed to guide the ablative therapy of cardiac arrhythmias. In this process both the site of origin of the arrhythmia and current pulses delivered from the tip of a catheter are modeled with a single equivalent moving dipole (SEMD) model in an infinite homogeneous volume conductor. However, because of lack of accurate torso geometry, the algorithm introduces systematic error in the estimated compared to the true dipole position. Computer simulations and experiments using a homogeneous saline tank have shown that this systematic error has minor effect in guiding the tip of the catheter to the site of the origin of the arrhythmia. In this study, to further investigate the accuracy of this method, we conducted experiments using a heterogeneous volume-conductor tank model. Our results demonstrate that the final distance between the catheter tip and the target site is about $3 \mathrm{~mm}$ and thus the lack of accurate torso geometry in the inverse problem appears to have minor effect in guiding the catheter.
\end{abstract}

\section{Introduction}

We have reported an inverse algorithm that allows the identification of bio-electrical sources by fitting potentials due to an arbitrary bio-electric source to a single equivalent moving dipole (SEMD) model [1-3]. In this algorithm, we achieve fast identification of the SEMD parameters, by employing a dipole model in an infinite homogeneous volume conductor and ignoring distortions due to the bounded, heterogeneous volume conductor. We have observed that the lack of accurate body geometry introduces a steady offset of the estimated compared to the true dipole position [1-3]. However, computer simulations using a realistic anatomic geometry torso model as well as homogeneous volume conductor tank experiments have demonstrated that the effect of the lack of accurate torso geometry in guiding the ablation catheter to the site of the origin of the arrhythmia is minor $[4,5]$. In this study, to further investigate the accuracy of this algorithm, we employed an inhomogeneous volumeconductor model in which a swine heart and two cylindrical acrylic tubes that simulated the lungs were placed in a tank that was filled with $0.9 \%$ saline solution.

\section{Methods}

\subsection{Inverse algorithm}

The inverse algorithm employs a SEMD model embedded in an infinite, homogeneous volume conductor [1-3]. The potential $\phi^{i}$ at position $\mathbf{r}^{i}$ on the surface of the tank due to a dipole at $\mathbf{r}$ ' with moment $\mathbf{p}$ is calculated by:

$$
\phi^{i}\left(\mathbf{r}^{\prime}, \mathbf{p}\right)=\frac{\mathbf{p} \bullet\left(\mathbf{r}^{i}-\mathbf{r}^{\prime}\right)}{\left|\mathbf{r}^{i}-\mathbf{r}^{\prime}\right|^{3}}
$$

The $\left(\mathbf{r}^{\prime}, \mathbf{p}\right)$ estimates are obtained by the simplex method that minimizes the $\chi^{2} / d o f$. Here, dof denotes the degrees of freedom, given by $N-6$, where $N$ is the number of the electrodes and $\chi^{2}$ is defined as:

$$
\chi^{2}=\sum_{i=1}^{N} \frac{\left(\phi^{i}-\phi_{m}^{i}\right)}{\sigma^{i}},
$$

where $\phi_{m}^{i}$ is the body surface potential at location $i$ due to the dipole and $\sigma^{i}$ is an estimate of the electrode noise at location $i$.

\subsection{Catheter guiding algorithm}

We have previously developed a method to evaluate our ability to guide a pair of electrodes (that simulate the tip of the ablation catheter) to a hypothetical target site (that simulates the site of the origin of the arrhythmia) $[4,5]$. To achieve that we place a pair of electrodes at the 
tip of a catheter and generate a dipole by applying current pulses through the electrodes at the catheter tip. We then use the same inverse algorithm to estimate both the SEMD due to the catheter tip and the dipole due to the target site. For faster computation, the algorithm estimates the SEMD (either due to the catheter tip or the target site) position and orientation by ignoring tissue inhomogeneities and realistic anatomic geometry as well as boundary effects. Thus, again it is expected that the SEMD estimates will suffer from systematic error. However, the effect of the systematic error is expected to progressively decrease as the dipole due to the catheter tip approaches the dipole due to the target site, i.e., the two dipoles coincide.

Thus, in the guiding process, we are trying to superpose the dipole due to the catheter tip to the dipole due to the target site. Firstly, the SEMD parameters due to the target site are estimated from potentials generated by current pulses delivered at that site and recorded at the surface of the tank. Then, the catheter is introduced into the appropriate cardiac chamber and the SEMD parameters due to the catheter tip are estimated by application of the inverse algorithm to potentials generated by current pulses delivered at the tip of the catheter. At each step the catheter tip is moved towards the target site by the amount of the estimated distance between the two dipoles (one due to the catheter tip and one due to the target site), after the dipole at the tip of the catheter is rotated so that the orientations of the dipoles are the same. These steps are repeated until the estimated distance between the two dipoles becomes less than a predetermined distance in two consecutive movements.

\subsection{Experimental methods}

We used a cylindrical tank (of radius $30 \mathrm{~cm}$ ) filled with $0.9 \%$ saline solution [5]. To introduce inhomogeneities, we placed in the tank a swine heart and two cylindrical acrylic tubes that were regarded as the lungs. The upper part of the heart was removed so that the catheter-tip that was mounted to a robotic manipulator could be inserted into the heart and accurately guided to the target site. Potentials generated by delivering current pulses at the catheter-tip and target sites in the heart were separately measured from 50 electrodes on the tank wall. Of these electrodes, 32 were distributed equally (8 columns $x 4$ lines) on the wall and the remaining 18 were placed near the heart. The current pulses were adjusted so that the maximum potential at the electrodes to be up to 5 $\mathrm{mV}$.

Figure 1 shows the experimental setup. A target dipole was placed in the heart and its SEMD parameters were estimated using the inverse algorithm from potentials recorded at the 50 electrodes on the tank wall. Another dipole at the catheter tip was introduced and its parameters were obtained in the same way. Then the tip on the manipulator was manually rotated and moved towards the target. In sequential movements, we attempted to superpose the dipole due to catheter-tip to the target until the estimated distance between the two sites became less than $2 \mathrm{~mm}$ in two consecutive movements. We carried out ten trials, starting from a random initial position with a random dipole orientation and at each one of them the dipole due to the catheter tip was guided towards a different target site dipole that had a different orientation. We also carried out another ten trials without the swine heart. In what follows, we will refer to this model, that does not include the heart, as the two-compartment model.

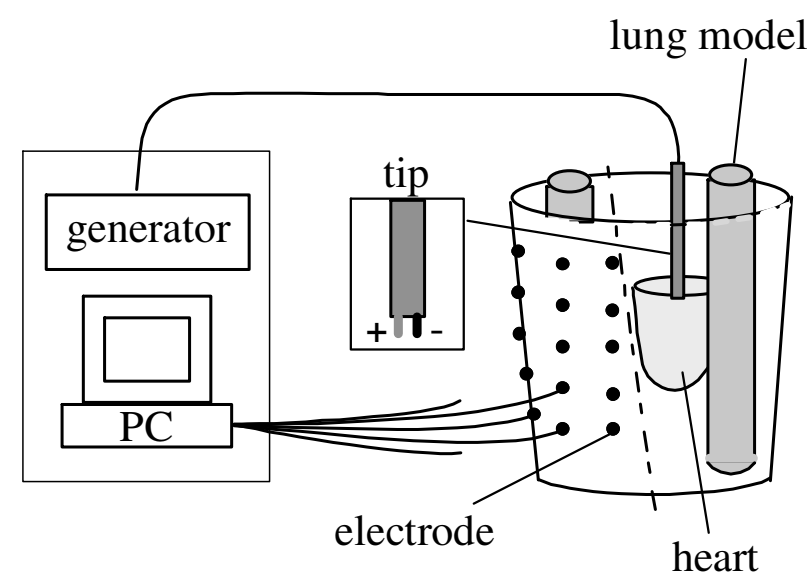

Figure 1 Experimental setup. A swine heart and two acrylic tubes that simulated the lungs were placed in a cylindrical tank filled with a $0.9 \%$ saline solution. Potential measurements due to dipoles at the catheter tip and the target site were made from 50 electrodes embedded on the wall of the tank.

We also investigated the accuracy of the guiding process in the possibility that under realistic conditions, i.e. in the time constraints of a clinical setting, it is likely, that one cannot accurately obtain the electrode locations at the body surface. Consequently, it would be desirable that the guiding procedure can be accurately performed without the precise electrode locations. For example, if patients with similar body size use the same electrode locations without diminishing the accuracy of the catheter guiding process, one does not have to repeat obtaining the electrode locations. This leads to an idea of standardized electrode locations for patients with similar body size. In this case, the positional uncertainty in the electrode location can be divided into: random (e.g. error in measuring the electrode locations) and systematic components. The latter corresponds to a case in which the patient's body size is different from the "standard" size.

Because in the homogeneous tank study [5], we have 
investigated the effect of the random component of the electrode positional uncertainty, in this study, we focused on the effect of the systematic component. We carried out trials by adding positional uncertainty at the electrode locations. For the sake of simplicity, the positional uncertainty in the electrode location was introduced only in the $\mathrm{x}$-axis (the anterior-posterior direction). In these trials, the $\mathrm{x}$-coordinate of each electrode location was multiplied by a factor of 0.7 or 1.2. These conditions corresponded to the cases in which the patient's body size was larger and smaller than the "standard" body size. Thus, while in the forward problem the potentials due to each of the dipoles were measured at the exact electrode locations, in the inverse problem the positional uncertainty in the electrode location was introduced to study its effect in the catheter guiding process to the target site.

\section{Results and discussion}

An example of the trajectory of the catheter tip in a guiding trial is illustrated in Figure 2. As depicted in the figure, in sequential steps, the tip gradually reached the target site. We should emphasize that the target site here indicates the true location of the target and not the one would estimate using the inverse algorithm.

The distance between the dipole due to the catheter tip and the dipole due to the target site using the threecompartment model is plotted against the iteration number in Figure 3. The summary results of the guiding trials employing the two- and three-compartment models are shown in Table 1. As shown in Table 1, in all ten trials, the tip of the catheter reached the target site within six movements. In the three-compartment model, the final distance ranged between 1.96 and $3.54 \mathrm{~mm}$, while in the two-compartment model the final distance ranged between 0.98 and $2.20 \mathrm{~mm}$. In the two-compartment model, the final distance and relative angle between the dipole due to the target and the dipole due to the catheter tip were smaller compared with those in the threecompartment model. This is because in the former model the degree of inhomogeneities was smaller when estimating the forward problem. However, in both models, the guiding process was terminated, on average, in five sequential steps.

The addition of the positional uncertainty in the electrode location slightly increased the final distance between the dipole due to the target and the dipole due to the catheter tip using the three-compartment model (compare Tables 1 and 2). The minimum and maximum distances were respectively 1.72 and $4.47 \mathrm{~mm}$ in the trials with a factor of 0.7 and 1.99 and $4.22 \mathrm{~mm}$ respectively in the trials with a factor of 1.2.

These results suggest that the lack of accurate torso geometry and inhomogeneities in tissue conductivity may have minor effect in the accuracy of guiding the tip of the catheter to the site of the origin of the arrhythmia.

We have also found that the positional uncertainty in the electrode location did not significantly affect the guiding process, suggesting the potential utility of using standardized electrode locations for similar body sizes. However, the larger average distance between the final location of the dipole due to the catheter tip and the dipole due to the target site suggests that the positional uncertainty diminishes the accuracy of the catheter guiding process when the patient's body size is very different from the "standard" one.

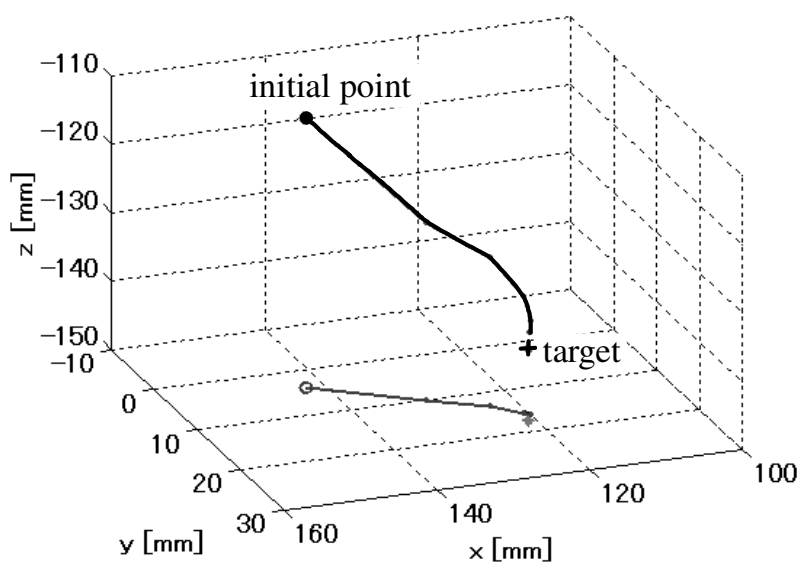

Figure 2 An example of a trajectory of the catheter tip. The grey line is the projection of the trajectory onto the $\mathrm{x}$ $y$ plane. In sequential steps, the catheter tip gradually approached to the target site.

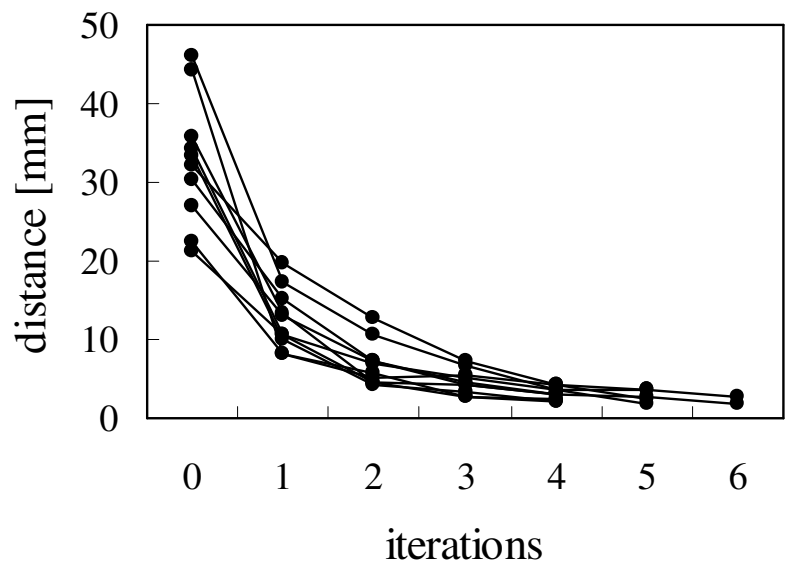

Figure 3 Changes in the distance between the target and the catheter tip in ten guiding trials using the threecompartment model. In all ten trials, the catheter tip reached the target site within six movements. 
Table 1 Experimental results (mean \pm stdev) for the (i) lungs (two-compartement), and (ii) lungs and heart (three-compartement) models

\begin{tabular}{ccc}
\hline model & $\begin{array}{c}\text { distance } \\
(\mathrm{mm})\end{array}$ & iterations \\
\hline lungs & $1.53 \pm 0.39$ & $4.8 \pm 0.79$ \\
\hline $\begin{array}{c}\text { lungs \& } \\
\text { heart }\end{array}$ & $2.66 \pm 0.52$ & $4.90 \pm 0.87$ \\
\hline
\end{tabular}

Table 2 Experimental results (mean \pm stdev) with positional uncertainty added in the electrode locations

\begin{tabular}{ccc}
\hline $\begin{array}{c}\text { positional } \\
\text { uncertainty }\end{array}$ & $\begin{array}{c}\text { distance } \\
(\mathrm{mm})\end{array}$ & iterations \\
\hline x 0.7 & $3.20 \pm 0.93$ & $5.11 \pm 0.60$ \\
\hline x 1.2 & $3.30 \pm 0.63$ & $4.72 \pm 0.78$ \\
\hline
\end{tabular}

\section{Concluding remarks}

In a saline tank study we investigated the accuracy of a method for guiding the ablative therapy of cardiac arrhythmias. We have found that the systematic error had a minor effect in the guiding process and, when the systematic error was the largest, the mean final distance between the catheter tip and the target site was less than 5 $\mathrm{mm}$. Since the accuracy required in a clinical setting is about $5 \mathrm{~mm}$, these results suggest that the lack of accurate torso geometry and tissue inhomogeneities as well as the inability to accurately measure the electrode locations may have minor effect in guiding the tip of the catheter to the site of the origin of the arrhythmia.

Since, the results presented in this study refer to the true location of the target site and not the one would estimate using the inverse algorithm, they are particularly important since the reported distance of the catheter to the target site reflects the total error in guiding the tip of the catheter to the target site.

In future studies, we aim to develop a catheter guiding system, which can be used in a clinical setting.

\section{Acknowledgements}

This work was partly supported by an American Heart Association Beginning Grant-in-Aid to AAA (\#0365304U).

\section{References}

[1] Armoundas AA, Feldman AB, Sherman DS, Cohen RJ. Applicability of the single equivalent dipole model to represent a spatially distributed bio-electrical source. Med. Biol. Eng. Comput., 2001;39:562-70.

[2] Armoundas AA, Feldman AB, Mukkamala R, Cohen RJ. A single equivalent moving dipole model: an efficient approach for localizing sites of origin of ventricular electrical activation. Ann. Biomed. Eng., 2003;31:564-76.

[3] Armoundas AA, Feldman AB, Mukkamala R, He B, Mullen TJ, Belk PA, Lee YZ, Cohen RJ. Statistical accuracy of a moving equivalent dipole model to identify sites of origin of cardiac electrical activation. IEEE Trans. Biomed. Eng., 2003;50:1360-70.

[4] Fukuoka Y, Oostendorp TF, Cohen RJ, Armoundas AA. A simulation study on a catheter navigation method for guiding the ablative therapy of cardiac arrhythmias. Proc. 23rd Ann. Conf. IEEE EMBS, 2001;CD-ROM:749.pdf.

[5] Shimojo T, Fukuoka Y, Minamitani1 H, Armoundas AA. A catheter guiding method for ablative therapy of cardiac arrhythmias by application of an inverse solution to body surface electrocardiographic signals. Proc. IEEE EMBS Asian Pacific Conf. Biomed. Eng., 2003;CDROM:020696-1.pdf

Address for correspondence:

Yutaka Fukuoka, Ph.D

Laboratory for Biosystems Modeling, School of Biomedical Science, Tokyo Medical and Dental University

2-3-10 Kandasurugadai, Chiyoda-ku, Tokyo 101-0062, Japan

Emai: fukuoka.bmi@tmd.ac.jp

or

Antonis A Armoundas, $\mathrm{PhD}$

Massachusetts General Hospital

Cardiovascular Research Center $14913^{\text {th }}$ Street

Charlestown, MA 02129, USA

Email: aarmoundas@partners.org 